\title{
Evaluation of serum inflammatory biomarkers as predictors of treatment outcome in pulmonary tuberculosis
}

\author{
A. Singanayagam ${ }^{\star}$, K. Manalan ${ }^{*}$, D. W. Connell ${ }^{\star}, \dagger$, J. D. Chalmers ${ }^{\ddagger}$, S. Sridhar ${ }^{\star}, \dagger$, A. I. \\ Ritchie $^{\star}$, A. Lalvani ${ }^{*}, \dagger$, M. Wickremasinghe ${ }^{*}$, and O. M. Kon ${ }^{\star}$ \\ "Chest and Allergy Department, St Mary's Hospital, Imperial College NHS Trust, London \\ †Tuberculosis Immunology Group, Imperial College London, London \\ ‡Tayside Respiratory Research Group, University of Dundee, Dundee, UK
}

\section{Summary}

Objective-To evaluate C-reactive protein (CRP), globulin and white blood cell (WBC) count as predictors of treatment outcome in pulmonary tuberculosis (PTB).

Methods-An observational study of patients with active PTB was conducted at a tertiary centre. All patients had serum CRP, globulin and WBC measured at baseline and at 2 months following commencement of treatment. The outcome of interest was requirement for extension of treatment beyond 6 months.

Results-There were 226 patients included in the study. Serum globulin $>45 \mathrm{~g} / \mathrm{l}$ was the only baseline biomarker evaluated that independently predicted requirement for treatment extension (OR 3.42, 95\%CI 1.59-7.32, $P<0.001$ ). An elevated globulin level that failed to normalise at 2 months was also associated with increased requirement for treatment extension (63.9\% vs. $5.1 \%, P$ $<0.001)$, and had a low negative likelihood ratio $(0.07)$ for exclusion of requirement for treatment extension. On multivariable analysis, an elevated globulin that failed to normalise at 2 months was independently associated with requirement for treatment extension (OR 6.13, 95\% CI 2.23-16.80, $P<0.001)$.

Conclusions-Serum globulin independently predicts requirement for treatment extension in PTB and outperforms CRP and WBC as a predictive biomarker. Normalisation of globulin at 2 months following treatment commencement is associated with low risk of requirement for treatment extension.

Correspondence to: Aran Singanayagam, Chest and Allergy Department, Imperial College London, London, UK. aransinga@gmail.com.

Conflicts of interest: AS has received honoraria for speaking from GlaxoSmithKline (Brentford, UK); JDC has received honoraria for speaking from Bayer (Leverkusen, Germany), Grifols (Barcelona, Spain), AstraZeneca (London, UK), GlaxoSmithKline, Pfizer (New York, NY, USA) and Napp (Cambridge, UK); AL has several patents on T-cell-based diagnosis. The ESAT-6/CFP-10 (early secretory antigenic target 6-culture filtrate protein 10) interferon-gamma ELISpot was commercialised by an Oxford University spin-out company (T-SPOT.TB, Oxford Immunotec, Abingdon, UK), in which Oxford University and AL have minority shares of equity and entitlement to royalties; OMK has chaired an advisory board for Janssen (Beerse, Belgium) and spoken on postgraduate educational sessions for Janssen and Otsuka Pharmaceuticals (Tokyo, Japan) at the European Respiratory Society. All other authors report no conflicts of interest. 


\section{Keywords}

biomarker; tuberculosis; outcome

TUBERCULOSIS (TB) IS A MAJOR public health concern and a leading cause of morbidity and mortality worldwide.1 Active pulmonary TB (PTB) is typically treated with an intensive phase of four antimicrobial agents for 2 months, followed by two agents during the 4-month continuation phase. This regimen leads to complete microbiological and clinical cure in the majority of cases.1,2 However, in some patients, routine treatment fails to adequately control and treat the disease, leading to failure of symptomatic improvement, prolonged infectivity and requirement for extension of therapy. 3 The length of antituberculosis treatment can have negative implications for patient adherence, and places increased pressure on health care systems. 4

Early evaluation of the response to anti-tuberculosis treatment has the potential to optimise routine clinical management of the disease and thus lead to improved outcomes. A biomarker that is predictive of likely response before treatment initiation or one that can be used to monitor subsequent treatment response could be invaluable to clinicians. Biomarkers measured at baseline could potentially identify patients with higher bacterial burden and/or enhanced inflammatory response who require more intensive monitoring and longer treatment regimens than patients with more minimal uncomplicated disease.5 Early treatment markers may allow the identification of patients in whom ineffective treatment has led to uncontrolled bacterial replication and development of drug resistance.5,6 Stratification of patients with TB at diagnosis or early in treatment into those requiring different therapeutic regimens and durations could improve adherence and treatment outcome and allow health care services to focus more on patients at greater risk of adverse treatment outcomes.7 An accurate predictive biomarker would also be invaluable in validating new TB drug candidates, thereby accelerating the development of novel therapies.

Currently available baseline markers of disease severity include chest radiography (CXR) findings8-10 and sputum smear grade, 9,11 and available clinical indicators of treatment response include symptomatic improvement, 12 weight gain, 13 resolution on CXR8 and sputum culture conversion.10,14 However, the results of microbiological tests can often be delayed and CXR assessment can be difficult to standardise and is complicated by the presence of chronic changes.5,6 A reliable marker that can be easily measured in serum as an accurate surrogate of treatment success is particularly desirable.

A number of immune parameters in serum have been shown to correlate with extent of disease and/or treatment response, including neopterin,15,16 C-reactive protein (CRP)17-19 and haematological parameters such as white blood cell (WBC) count and erythrocyte sedimentation rate.20,21 However, these parameters have only been assessed in small studies at the onset of disease. Globulins are a collection of proteins that can be readily measured in the blood. Total globulin levels are routinely measured in serum samples and are non-specifically elevated in response to several inflammatory conditions, including active TB.22 Studies have previously shown that globulin levels in serum correlate with adverse outcomes from Pneumocystis jirovecii pneumonia23 and lung cancer.24 The value 
of serum globulin as a predictor of outcome in TB has not been formally evaluated previously.

The aim of the present study was to assess the value of measuring serum levels of routine inflammatory biomarkers, globulin, CRP and WBC at baseline and 2 months following treatment commencement to predict outcome in patients treated for active PTB.

\section{Methods}

\section{Study population}

We conducted an observational study of consecutive adult patients aged $>16$ years with active bacteriologically confirmed PTB commenced on anti-tuberculosis chemotherapy at St Mary's Hospital, London, UK, between January 2008 and January 2013. The study received local approval from St Mary's Hospital. Patients were included if they had sputum or bronchoalveolar lavage samples that were positive for culture of Mycobacterium tuberculosis.

Exclusion criteria were 1) patients who were treated based on clinical likelihood for PTB but without evidence of positive culture for M. tuberculosis; and 2) loss to follow-up or failure to complete treatment.

\section{Measurement of inflammatory biomarkers in serum}

All patients included in the study had measurement of CRP, WBC and total globulin levels in serum samples taken at baseline before initiating anti-tuberculosis treatment, with repeat measurements undertaken at 2 months following commencement of treatment. The normal ranges of the assays were as follows: CRP 0-10 mg/l, globulin 19-35 g/l, WBC count 4.0$11.0 \times 10^{9}$ cells/1.

\section{Microbiological evaluation}

Microscopy was performed in all patients who produced sputum or underwent bronchoscopy with bronchoalveolar lavage (BAL). The density of acid-fast bacilli was graded as scanty, 1 , 2 or $3+$ according to standard protocols. 25 TB culture was performed by the incubation of sputum or BAL samples using the BACTEC ${ }^{\text {TM }}$ MGIT ${ }^{\text {Tм }} 960$ system (BD, Franklin Lakes, NJ, USA) for up to 6 weeks.

\section{Radiographic evaluation}

As part of the initial diagnostic evaluation, all patients included in the study underwent standard posteroanterior CXR to assess for signs of active TB, including nodules, consolidation and cavitation.

\section{Outcome}

The outcome of interest was requirement for extension of anti-tuberculosis treatment beyond 6 months. Indications for extension of treatment were left to the discretion of the treating physician, and included one or more of the following factors: persistent smear or culture positivity, failure of improvement on CXR, drug resistance, persistent symptoms, poor 
adherence to treatment, presence of extra-pulmonary disease and drug-induced liver injury. We also conducted a separate analysis to evaluate the outcome of persistent sputum smear and/or culture positivity (defined as $>2$ months following treatment initiation).

\section{Statistical analysis}

All data were analysed using SPSS, version 13.0 for windows (Statistical Package for the Social Sciences, Chicago, IL, USA). The $\chi^{2}$ test was used to compare categorical variables. The Mann-Whitney $U$-test and the Kruskal Wallis test were used to compare continuous variables between two or multiple groups, respectively. Sensitivity, specificity, positive (PPV) and negative predictive values (NPV), positive (LR+) and negative likelihood ratios (LR-) and area under the receiver operator characteristic curve (AUC) were used to assess the value of serum biomarkers for the prediction of outcomes of interest.

We used multivariable logistic regression to evaluate the association of baseline and 2-month levels of globulin, CRP and WBC count with outcomes of interest. The following variables were included in the regression model: age $>50$ years, male sex, requirement for directly observed treatment, alcohol excess, human immunodeficiency virus (HIV) infection, drug resistance, smear positivity, poor adherence, cavitating disease and multilobar CXR changes. A two-tailed $P<0.05$ was considered statistically significant.

\section{Results}

There were 226 patients included in the study. Baseline demographics of the study cohort are summarised in Table 1.

\section{Correlation of pre-treatment globulin levels with microbiological and radiological disease burden}

Measurement of inflammatory biomarkers prior to the commencement of anti-tuberculosis treatment identified 175 patients $(77.4 \%)$ with an elevated serum globulin (>35 g/dl), 155 patients $(68.6 \%)$ with elevated serum CRP $(>10 \mathrm{mg} / \mathrm{l})$ and 28 patients $(12.4 \%)$ with an elevated WBC count $\left(>11.0 \times 10^{9} / \mathrm{l}\right)$. The correlation of pre-treatment levels of these biomarkers with microbiological and radiological markers of disease burden is shown in the Figure, including smear positivity (Figure A-C), radiographic lobar involvement (Figure DF) and presence of cavitary disease (Figure G-I).

\section{Predictive value of pre-treatment serum inflammatory biomarkers for requirement of therapy extension}

The value of pre-treatment serum CRP, globulin and WBC count levels in predicting the requirement for extension of anti-tuberculosis treatment (.6 months) was evaluated. Reasons for treatment extension are shown in Appendix Table A.1.* Higher levels of serum globulin, CRP and WBC count were all significantly associated with increased frequency of requirement for treatment extension (Table 2).

The sensitivity, specificity, PPV and NPV, LR+ and LR- and AUCs for pre-treatment globulin $>45 \mathrm{~g} / \mathrm{l}, \mathrm{CRP}>50 \mathrm{mg} / \mathrm{dl}$ and $\mathrm{WBC}$ count $>11 \times 10^{9} / \mathrm{l}$ with regard to the prediction 
of requirement for treatment extension were evaluated. All tests had poor to moderate predictive value, with globulin having the highest AUC (0.70; Table 3).

\section{Multivariable analyses}

On multivariable analysis, pre-treatment globulin $>45 \mathrm{~g} / \mathrm{l}$ was independently associated with the requirement for treatment extension (odds ratio [OR] 3.42, 95\% confidence interval [CI] $1.59-7.32, P<0.001)$. Pre-treatment CRP $>50 \mathrm{mg} / \mathrm{l}$ and $\mathrm{WBC}$ count $>11 \times 10^{9} / 1$ were not independently associated with treatment extension (Appendix Table A.2).

\section{Evaluation of serial inflammatory biomarker measurements for the prediction of treatment outcome in pulmonary tuberculosis}

Having investigated the predictive value of pre-treatment levels of inflammatory biomarkers, we next evaluated whether measurement of repeat biomarker levels at 2 months following treatment initiation could predict treatment outcome. The rates of requirement for treatment extension stratified according to whether or not the levels of CRP, globulin or WBC count normalised at 2 months are shown in Table 2. Significantly increased rates of requirement for treatment extension were observed in patients in whom globulin or CRP failed to normalise by 2 months after initiation of treatment; however, no significant association was observed for normalisation of WBC count (Table 2).

We then formally assessed the predictive value of normalisation of globulin, CRP and WBC count at the 2-month measurement to determine persistent smear and/or culture positivity and requirement for treatment extension. Globulin levels that normalised at 2 months had a LR- of 0.07 in excluding requirement for treatment extension (Table 3), indicating that this represents a clinically valuable rule-out test.26 CRP and WBC count had poor LR- for excluding requirement for treatment extension.

\section{Multivariable analysis}

On multivariable analysis, an elevated globulin level that failed to normalise by 2 months was independently associated with the requirement for treatment extension (OR 6.13, 95\% CI 2.23-16.8, $P<0.001)$. CRP that failed to normalise was also independently associated with treatment extension (OR 3.0, 95\%CI 1.15-7.82, $P=0.025$; Appendix Table A.2). An analysis of WBC count normalisation could not be carried out due to the small number of patients with elevated baseline levels.

\section{Subgroup analysis of baseline and serial biomarkers for the prediction of treatment extension associated with persistent smear or culture positivity or failure of radiographic improvement}

In addition to the evaluation of inflammatory biomarkers as predictors of treatment extension, we also carried out a subanalysis to evaluate these tests for the prediction of surrogate markers of treatment response, persistent 2-month sputum smear or culture positivity and failure of CXR improvement. Increasing pre-treatment levels of all three biomarkers correlated significantly with increased frequency of treatment extension associated with failure of CXR improvement, but not with persistent smear and/or culture positivity (Table 2). Significantly increased rates of persistent smear and/or culture positivity 
were observed in patients in whom globulin, CRP or WBC count did not normalise by 2 months after initiation of treatment. Patients in whom globulin or CRP did not normalise also had increased rates of treatment extension due to failure of CXR improvement (Table 2). Similar to the outcome of requirement for treatment extension, globulin levels that normalised at 2 months also had the lowest LR-for excluding treatment extension associated with persistent smear or culture positivity or failure of CXR improvement (Table $3)$.

\section{Discussion}

We evaluated the predictive value of routinely measured serum biomarkers, CRP and globulin levels and WBC count for the prediction of treatment outcome in patients treated for active PTB. We found that baseline pre-treatment levels of all three biomarkers correlated with the extent of radiological and microbiological disease burden, and higher pre-treatment biomarker levels were associated with increased frequency of requirement for treatment extension. However, after correction for other potential confounding variables, globulin $>45 \mathrm{~g} / \mathrm{l}$ was the only baseline biomarker found to be independently associated with treatment outcome.

All of the tests evaluated performed poorly as pre-treatment predictors of the clinically relevant outcome (requirement for treatment extension), with AUC values $<0.7$, the threshold that represents a clinically useful test. This suggests that none of these tests could be used alone to accurately predict treatment outcome at baseline. Of the three markers evaluated, pre-treatment globulin level had the highest AUC value as baseline predictor of outcome. In particular, only 28 patients (12.3\%) had an elevated WBC count before treatment initiation, which suggests that it is extremely unlikely to be clinically useful as a predictive biomarker. This was reflected in the low AUC value of 0.58. It is perhaps unsurprising that biomarkers were poorly predictive of length of treatment. This outcome is not solely dependent on mycobacterial burden or inflammatory response, which would be expected to correlate directly with serum levels of immune markers such as globulin, but may also be determined by other unrelated factors such as poor treatment adherence or complications such as drug-induced liver injury.

In addition to assessing the value of pre-treatment biomarker levels, we also evaluated the predictive value of repeat measurement of inflammatory biomarkers at 2 months following treatment initiation to determine whether failure of normalisation of these markers correlated with the requirement for treatment extension. The failure of normalisation of globulin or CRP levels was independently associated with the requirement for treatment extension. However, globulin had the lowest LR- for excluding requirement for treatment extension. It is recognised that a threshold of $\mathrm{LR}<0.1$ is indicative of a clinically useful rule-out test.26 The low LR- of globulin normalisation at 2 months suggests that it is a good marker of response to adequate treatment. Our data therefore suggest that the measurement of globulin in patients commenced on anti-tuberculosis treatment, with its subsequent normalisation by 2 months, is associated with very low rates of the requirement for treatment extension; this leads us to believe that globulin may be a useful adjunct to clinical judgment in identifying 
low-risk patients. By contrast, 2-month CRP and WBC count measures had high LR-, thus suggesting their lack of utility in a clinical setting.

Our finding that globulin could predict the requirement for treatment extension in TB suggests that it could be a useful marker in clinical practice. Serum globulin is a simple, cheap and widely available blood test. In most centres, all patients with active TB are routinely reviewed at 2 months to assess treatment response and decide whether treatment can be moved from the intensive to the continuation phase. Our finding that the normalisation of globulin levels at 2 months can exclude requirement for treatment extension thus offers a predictive test that can be measured rapidly and reliably, without the need for additional hospital visits. In combination with other recognised markers of treatment response, including weight gain,13 symptomatic improvement12 and resolution of CXR changes, 8 serum globulin provides an additional clinical marker for treatment response that can be easily assessed by clinicians and could aid in making decisions about the safe and appropriate move to the continuation phase of treatment.

The length of anti-tuberculosis treatment is an important endpoint, as it may have negative implications for patient adherence.27 Historical data suggest that patients who respond early to treatment may be safely managed with a shortened course of antibiotic therapy, 28 although this remains controversial, and recent studies have reported worse outcomes for 4month regimens.29,30 A test such as the globulin test could help stratify patients into risk groups to guide duration of treatment and, possibly, improve adherence and outcomes and reduce treatment-related costs. Further studies are required to determine whether globulin, alone or in combination with other predictors, could be used in this way.

Total globulin level reflects a combination of specific proteins, including the alpha globulins such as alpha-1-antitrypsin and haptoglobulin, transferrin, complement and immunoglobulins. Previous studies have shown that complement C431 and M. tuberculosisspecific immunoglobulins32,33 are elevated in serum from patients with active TB. We did not formally carry out serum protein electrophoresis to identify subcomponents that were specifically elevated in patients with active TB, but data from previous studies offer a biologically plausible explanation for our finding that total globulin is elevated in patients with active TB and correlates with treatment outcomes. However, as our study was observational in nature, we could not perform all measurements in all patients. The study may also have been limited by the small sample size, as indicated by the wide confidence intervals observed in some of our analyses.

In conclusion, the measurement of paired serum globulin samples at baseline and 2 months into treatment can help identify patients at lower risk of requirement for treatment extension. Globulin outperformed the other biomarkers evaluated in our study. When combined with other clinical measures, globulin may provide clinicians with a simple and rapid means of identifying lower-risk patients. Whether globulin measures could be used to predict other more robust measures of treatment success, such as recurrent disease and TB-related death, remains unknown, and further studies in independent populations are required. 


\section{Appendix}

Refer to Web version on PubMed Central for supplementary material.

\section{Acknowledgements}

The study was undertaken at St Mary's Hospital, Imperial College Healthcare National Health Service Trust, which is supported by the National Institute of Health Research Biomedical Research Centre funding scheme.

\section{References}

1. World Health Organization. Global tuberculosis report, 2014. Geneva, Switzerland: WHO; 2014. WHO/HTM/TB/2014.08

2. Han T. Effectiveness of standard short-course chemotherapy for treating tuberculosis and the impact of drug resistance on its outcome. Int J Evid Based Healthc. 2006; 4:101-117. [PubMed: 21631761]

3. Mitruka K, Winston CA, Navin T R. Predictors of failure in timely tuberculosis treatment completion, United States. Int J Tuberc Lung Dis. 2012; 16:1075-1082. [PubMed: 22668774]

4. Kayigamba FR, Bakker MI, Mugisha V, et al. Adherence to tuberculosis treatment, sputum smear conversion and mortality: a retrospective cohort study in 48 Rwandan clinics. PLOS ONE. 2013; 8 e73501.

5. Walzl G, Ronacher K, Hanekom W, Scriba TJ, Zumla A. Immunological biomarkers of tuberculosis. Nature Rev Immunol. 2011; 11:343-354. [PubMed: 21475309]

6. Perrin FM, Lipman MC, McHugh TD, Gillespie SH. Biomarkers of treatment response in clinical trials of novel antituberculosis agents. Lancet Infect Dis. 2007; 7:481-490. [PubMed: 17524807]

7. Chirwa T, Nyasulu P, Chirwa E, et al. Levels of tuberculosis treatment adherence among sputum smear positive pulmonary tuberculosis patients attending care at Zomba Central hospital, southern Malawi, 2007-2008. PLOS ONE. 2013; 8 e63050.

8. Ralph AP, Ardian M, Wiguna A, et al. A simple, valid, numerical score for grading chest x-ray severity in adult smear-positive pulmonary tuberculosis. Thorax. 2010; 65:863-869. [PubMed: 20861290]

9. Hesseling AC, Walzl G, Enarson DA, et al. Baseline sputum time to detection predicts month two culture conversion and relapse in non-HIV-infected patients. Int J Tuberc Lung Dis. 2010; 14:560570. [PubMed: 20392348]

10. Benator D, Bhattacharya M, Bozeman L, et al. Rifapentine and isoniazid once a week versus rifampicin and isoniazid twice a week for treatment of drug-susceptible pulmonary tuberculosis in HIV-negative patients: a randomised clinical trial. Lancet. 2002; 360:528-534. [PubMed: 12241657]

11. Atif M, Sulaiman SA, Shafie AA, Ali I, Asif M, Babar ZU. Treatment outcome of new smear positive pulmonary tuberculosis patients in Penang, Malaysia. BMC Infect Dis. 2014; 14:399. [PubMed: 25037452]

12. Hales CM, Heilig CM, Chaisson R, et al. The association between symptoms and microbiologically defined response to tuberculosis treatment. Ann Am Thoracic Soc. 2013; 10:1825.

13. Krapp F, Veliz JC, Cornejo E, Gotuzzo E, Seas C. Bodyweight gain to predict treatment outcome in patients with pulmonary tuberculosis in Peru. Int J Tuberc Lung Dis. 2008; 12:1153-1159. [PubMed: 18812045]

14. Mitchison DA. Assessment of new sterilizing drugs for treating pulmonary tuberculosis by culture at 2 months. Am Rev Respir Dis. 1993; 147:1062-1063. [PubMed: 8466107]

15. Hosp M, Elliott AM, Raynes JG, et al. Neopterin, beta 2-microglobulin, and acute phase proteins in HIV-1-seropositive and -seronegative Zambian patients with tuberculosis. Lung. 1997; 175:265275. [PubMed: 9195554]

16. Immanuel C, Rajeswari R, Rahman F, Kumaran PP, Chandrasekaran V, Swamy R. Serial evaluation of serum neopterin in HIV seronegative patients treated for tuberculosis. Int J Tuberc Lung Dis. 2001; 5:185-190. [PubMed: 11258513] 
17. Djoba Siawaya JF, Bapela NB, Ronacher K, et al. Immune parameters as markers of tuberculosis extent of disease and early prediction of anti-tuberculosis chemotherapy response. J Infect. 2008; 56:340-347. [PubMed: 18359089]

18. Scott GM, Murphy PG, Gemidjioglu ME. Predicting deterioration of treated tuberculosis by corticosteroid reserve and C-reactive protein. J Infect. 1990; 21:61-69. [PubMed: 2384681]

19. Bajaj G, Rattan A, Ahmad P. Prognostic value of 'C' reactive protein in tuberculosis. Indian Pediatr. 1989; 26:1010-1013. [PubMed: 2630443]

20. Brahmbhatt S, Black GF, Carroll NM, et al. Immune markers measured before treatment predict outcome of intensive phase tuberculosis therapy. Clin Exp Immunol. 2006; 146:243-252. [PubMed: 17034576]

21. Morris CD, Bird AR, Nell H. The haematological and biochemical changes in severe pulmonary tuberculosis. Q J Med. 1989; 73:1151-1159. [PubMed: 2616737]

22. Moraes ML, Ramalho DM, Delogo KN, et al. Association of serum levels of iron, copper, and zinc, and inflammatory markers with bacteriological sputum conversion during tuberculosis treatment. Biol Trace Elem Res. 2014; 160:176-184. [PubMed: 24958018]

23. Ewig S, Bauer T, Schneider C, et al. Clinical characteristics and outcome of Pneumocystis carinii pneumonia in HIV-infected and otherwise immunosuppressed patients. Eur Respir J. 1995; 8:1548-1553. [PubMed: 8575583]

24. Yao Y, Zhao M, Yuan D, Gu X, Liu H, Song Y. Elevated pretreatment serum globulin albumin ratio predicts poor prognosis for advanced non-small cell lung cancer patients. J Thorac Dis. 2014; 6:1261-1270. [PubMed: 25276368]

25. Kelly PM, Ardian M, Waramori G, et al. A community-based TB drug susceptibility study in Mimika District, Papua Province, Indonesia. Int J Tuberc Lung Dis. 2006; 10:167-171. [PubMed: 16499255]

26. Deeks JJ, Altman DG. Diagnostic tests 4: likelihood ratios. BMJ. 2004; 329:168-169. [PubMed: 15258077]

27. Kruk ME, Schwalbe NR, Aguiar CA. Timing of default from tuberculosis treatment: a systematic review. Trop Med Int Health. 2008; 13:703-712. [PubMed: 18266783]

28. Hong Kong Chest Service/British Medical Research Council. Controlled trial of 2, 4, and 6 months of pyrazinamide in 6-month, three-times-weekly regimens for smear-positive pulmonary tuberculosis, including an assessment of a combined preparation of isoniazid, rifampin, and pyrazinamide. Results at 30 months. Am Rev Respir Dis. 1991; 143(4 Pt 1):700-706. [PubMed: 1901199]

29. Johnson JL, Hadad DJ, Dietze R, et al. Shortening treatment in adults with noncavitary tuberculosis and 2-month culture conversion. Am J Respir Crit Care Med. 2009; 180:558-563. [PubMed: 19542476]

30. Jindani A, Harrison TS, Nunn AJ, et al. High-dose rifapentine with moxifloxacin for pulmonary tuberculosis. N Engl J Med. 2014; 371:1599-1608. [PubMed: 25337749]

31. Wang C, Li YY, Li X, et al. Serum complement C4b, fibronectin, and prolidase are associated with the pathological changes of pulmonary tuberculosis. BMC Infect Dis. 2014; 14:52. [PubMed: 24484408]

32. Welch RJ, Lawless KM, Litwin CM. Antituberculosis IgG antibodies as a marker of active Mycobacterium tuberculosis disease. Clin Vaccine Immunol. 2012; 19:522-526. [PubMed: 22301692]

33. Ashenafi S, Aderaye G, Zewdie M, et al. BCG-specific IgG-secreting peripheral plasmablasts as a potential biomarker of active tuberculosis in HIV negative and HIV positive patients. Thorax. 2013; 68:269-276. [PubMed: 22923457] 


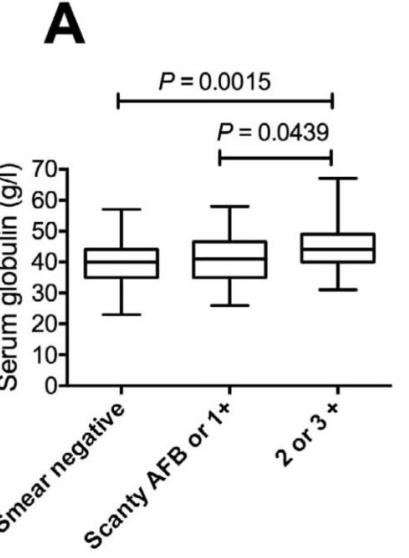

D
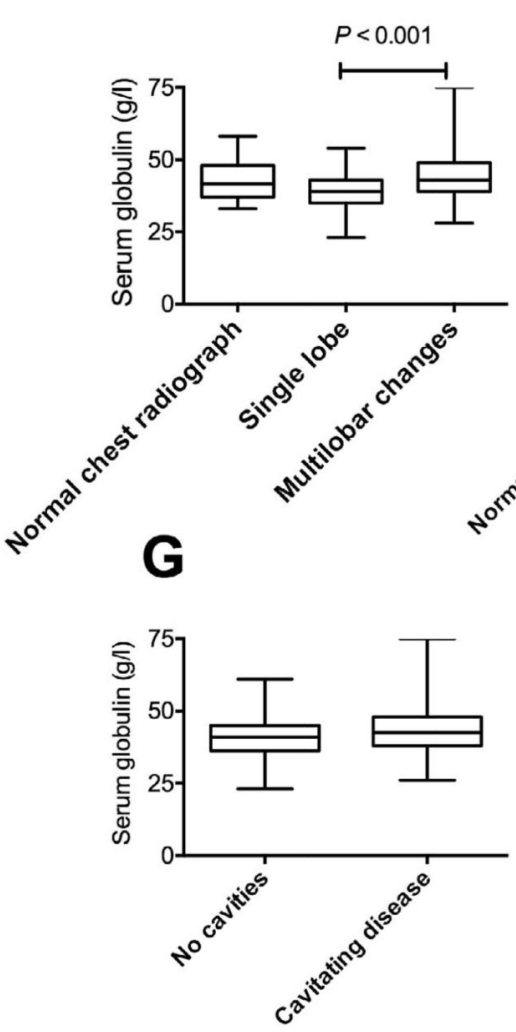
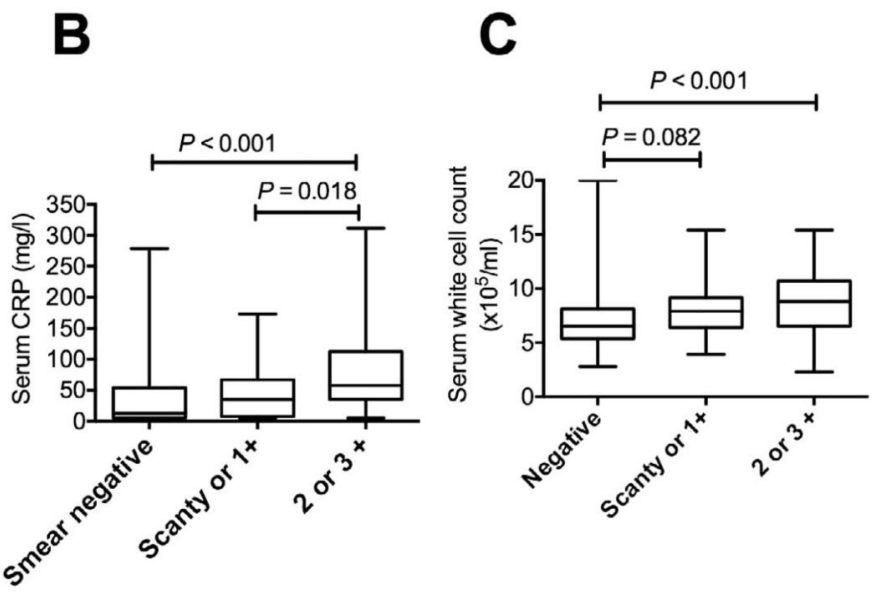

E
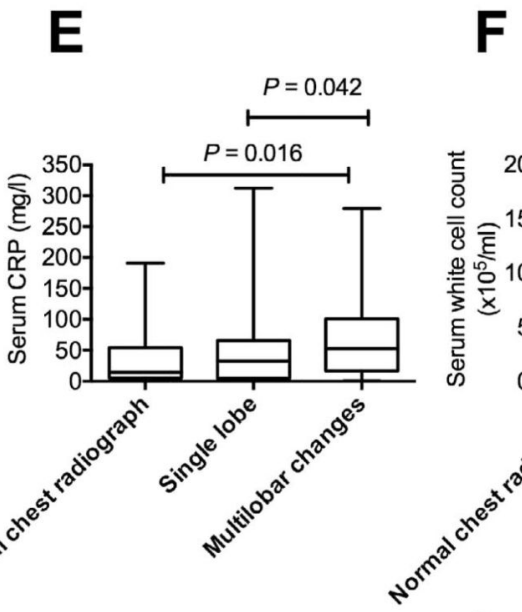

$\mathbf{H}$

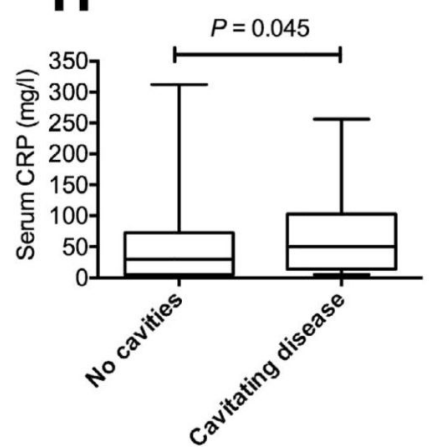

Figure.

Correlation between pre-treatment biomarker levels and microbiological and radiological markers of disease burden. Box and whisker plot showing median globulin, CRP and WBC count levels stratified according to A-C) smear grade, D-F) lobar involvement on CXR and G-I) presence of cavitating disease on CXR. Comparison of groups by Kruskal-Wallis test in A-F and Mann-Witney $U$ test in $\mathbf{G}-\mathbf{I}$. AFB = acid-fast bacilli; $\mathrm{CRP}=\mathrm{C}$-reactive protein; $\mathrm{WBC}=$ white blood cell; $\mathrm{CXR}=$ chest radiograph. 


\section{Table 1}

Baseline demographics of the study population

\begin{tabular}{|c|c|}
\hline Characteristic & $n(\%)$ \\
\hline \multicolumn{2}{|l|}{ Demographics } \\
\hline Age, years, median [IQR] & 33 [25.3-49] \\
\hline Male sex & $148(65.5)$ \\
\hline UK-born & $53(23.5)$ \\
\hline Caucasian & $55(24.3)$ \\
\hline Black African & $49(21.7)$ \\
\hline Asian & $51(22.6)$ \\
\hline Other & $71(31.4)$ \\
\hline \multicolumn{2}{|l|}{ Comorbidities } \\
\hline Chronic lung disease & $15(6.6)$ \\
\hline Diabetes mellitus & $6(2.7)$ \\
\hline Alcohol excess & $11(4.9)$ \\
\hline HIV & $7(3.1)$ \\
\hline Other immunosuppression & $2(0.9)$ \\
\hline Chronic renal failure & $2(0.9)$ \\
\hline Chronic liver disease & $2(0.9)$ \\
\hline Smoker & $21(9.3)$ \\
\hline \multicolumn{2}{|l|}{ Microbiology } \\
\hline Smear negative & $115(50.9)$ \\
\hline \multicolumn{2}{|l|}{ Smear grade } \\
\hline Scanty AFB & $32(14.2)$ \\
\hline+ & $18(8.0)$ \\
\hline++ & $18(8.0)$ \\
\hline+++ & $43(19.0)$ \\
\hline \multicolumn{2}{|c|}{ Persistent smear and/or culture positivity } \\
\hline (>60 days) & $20(8.9)$ \\
\hline Non-MDR-TB drug resistance & $19(8.4)$ \\
\hline MDR-TB & $9(4.0)$ \\
\hline \multicolumn{2}{|l|}{ Radiology } \\
\hline Normal chest radiograph & $35(15.5)$ \\
\hline Cavitating disease & $76(33.6)$ \\
\hline Multi-lobar changes & $78(34.5)$ \\
\hline Pleural effusion & $31(13.7)$ \\
\hline \multicolumn{2}{|l|}{ Treatment outcome } \\
\hline \multicolumn{2}{|c|}{ Requirement for extension of treatment } \\
\hline (>6 months) & $86(38.1)$ \\
\hline TB recurrence & $2(0.9)$ \\
\hline TB-related death & $2(0.9)$ \\
\hline
\end{tabular}

$\mathrm{IQR}=$ interquartile range; $\mathrm{HIV}=$ human immunodeficiency virus; $\mathrm{AFB}=$ acid-fast bacilli; $\mathrm{MDR}-\mathrm{TB}=$ multidrug-resistant tuberculosis. 
Table 2

Outcomes stratified according to pre-treatment and 2-month biomarker levels

\begin{tabular}{|c|c|c|c|c|}
\hline & $n$ & $\begin{array}{l}\text { Requirement for treatment extension } \\
\qquad n(\%)\end{array}$ & $\begin{array}{c}\text { Persistent smear and/or culture } \\
\text { positivity } \\
n(\%)\end{array}$ & $\begin{array}{l}\text { Failure of radiographic improvement } \\
\qquad n(\%)\end{array}$ \\
\hline \multicolumn{5}{|c|}{ Pre-treatment globulin, g/l } \\
\hline$<35$ & 51 & $10(19.6)$ & $4(7.8)$ & $3(5.9)$ \\
\hline $36-40$ & 56 & $17(30.4)$ & $4(7.1)$ & $4(7.1)$ \\
\hline $41-45$ & 57 & $19(33.3)$ & $5(8.8)$ & $9(15.8)$ \\
\hline $46-50$ & 42 & $24(57.1)$ & $4(9.5)$ & $12(28.6)$ \\
\hline$>50$ & 20 & $16(80.0)$ & $3(15.0)$ & $9(45)$ \\
\hline$P$ value & & $<0.001$ & 0.88 & $<0.002$ \\
\hline \multicolumn{5}{|c|}{ Globulin fails to normalise by 2 months } \\
\hline Yes & 97 & $72(74.2)$ & $12(12.4)$ & $27(27.8)$ \\
\hline No & 78 & $4(5.1)$ & $1(1.3)$ & $4(5.1)$ \\
\hline$P$ value & & $<0.001$ & 0.007 & $<0.001$ \\
\hline \multicolumn{5}{|c|}{ Pre-treatment CRP, mg/l } \\
\hline$<10$ & 71 & $14(19.7)$ & $3(4.2)$ & $8(11.3)$ \\
\hline $11-50$ & 70 & $27(38.6)$ & $5(7.1)$ & $9(12.9)$ \\
\hline $51-100$ & 45 & $21(46.7)$ & $4(8.9)$ & $7(15.6)$ \\
\hline $100-150$ & 21 & $14(66.7)$ & $4(19.0)$ & $6(28.6)$ \\
\hline$>150$ & 19 & $10(52.6)$ & $3(15.7)$ & $7(36.8)$ \\
\hline$P$ value & & $<0.001$ & 0.184 & 0.039 \\
\hline \multicolumn{5}{|c|}{ CRP fails to normalise by 2 months } \\
\hline Yes & 42 & $27(61.4)$ & $7(16.7)$ & $12(28.6)$ \\
\hline No & 113 & $44(28.9)$ & $4(3.5)$ & $9(8.0)$ \\
\hline$P$ value & & 0.006 & 0.0095 & 0.0037 \\
\hline \multicolumn{5}{|c|}{ Pre-treatment WBC count, $x 10^{9} / 1$} \\
\hline$<4.0$ & 10 & $2(20)$ & $1(10.0)$ & $1(10.0)$ \\
\hline $4-11$ & 188 & $66(35.1)$ & $14(7.4)$ & $26(13.8)$ \\
\hline $11-14$ & 18 & $11(61.1)$ & $3(16.7)$ & $6(33.3)$ \\
\hline$>14$ & 10 & $7(70)$ & $1(10.0)$ & $4(40.0)$ \\
\hline$P$ value & & 0.015 & 0.596 & 0.029 \\
\hline \multicolumn{5}{|c|}{ WBC count fails to normalise } \\
\hline Yes & 3 & $3(100)$ & $2(66.7)$ & $3(100)$ \\
\hline No & 25 & $14(56)$ & $2(8.0)$ & $19(76.0)$ \\
\hline$P$ value & & 0.258 & 0.045 & 1 \\
\hline
\end{tabular}

$\mathrm{CRP}=\mathrm{C}$-reactive protein $; \mathrm{WBC}=$ white blood cell. 


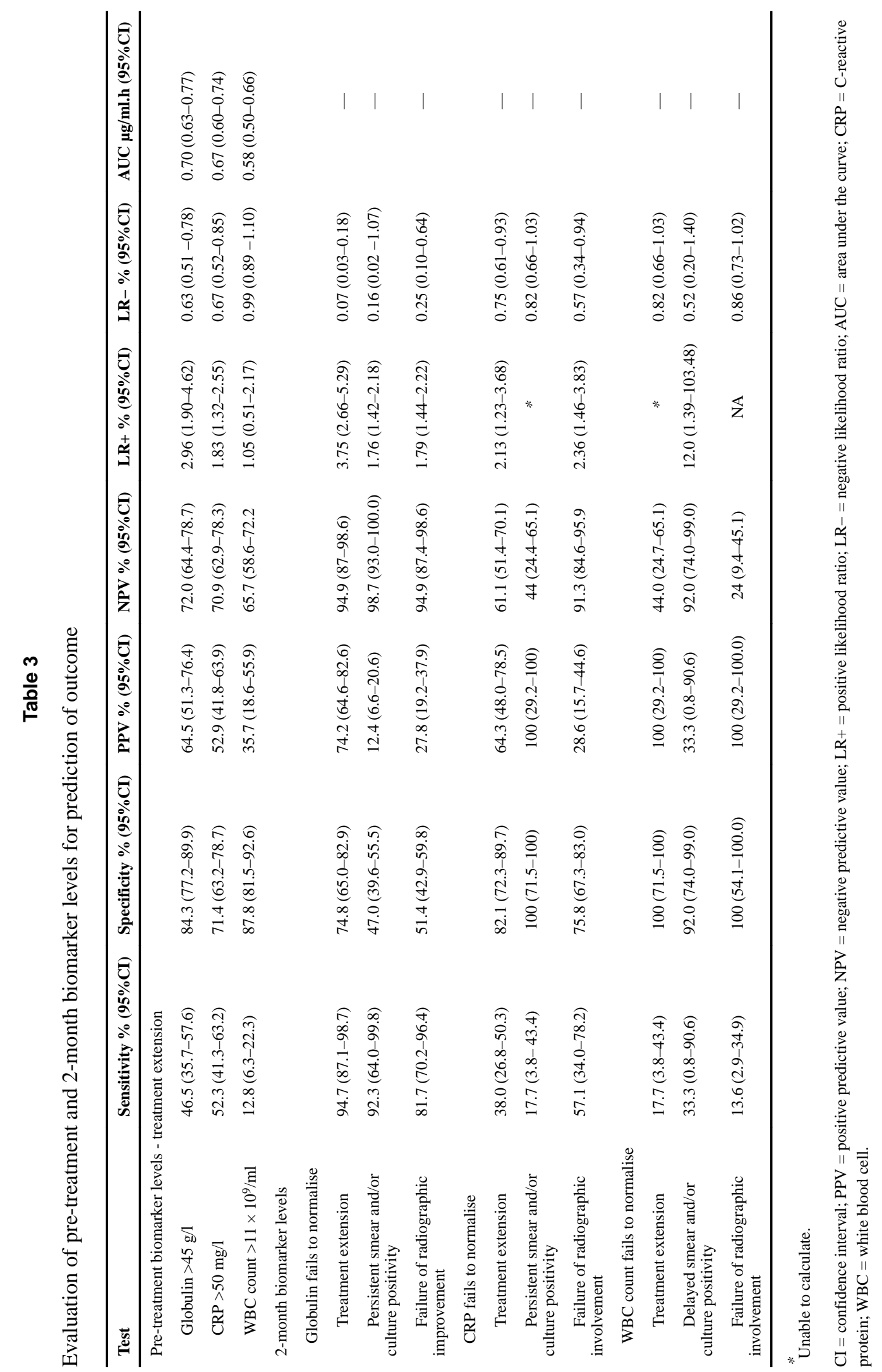

